\title{
Airfoil Optimization Using Practical Aerodynamic Design Requirements
}

\author{
Howard P. Buckley, ${ }^{*}$ Beckett Y. Zhou ${ }^{\dagger}$ and David W. Zingg ${ }^{\ddagger}$ \\ Institute for Aerospace Studies, University of Toronto \\ 4925 Dufferin St., Toronto, Ontario, M3H 5T6, Canada
}

\begin{abstract}
Practical aerodynamic design problems must balance the goal of performance optimization over a range of on-design operating conditions with the need to meet design constraints at various off-design operating conditions. Such design problems can be cast as multipoint optimization problems where the on-design and off-design operating conditions are represented as design points with corresponding objective/constraint functions. Two methods are presented for obtaining optimal airfoil designs that satisfy all design objectives and constraints. The first method uses an unconstrained optimization algorithm where the optimal design is achieved by minimizing a weighted sum of the objective functions at each of the operating conditions. To address the competing design objectives between on-design and off-design operating conditions, an automated procedure is used to efficiently weight the off-design objective functions so as to limit their influence on the overall optimization while satisfying the design constraints. The second method uses the constrained optimization algorithm SNOPT, which allows the aerodynamic constraints imposed at the off-design operating conditions to be treated explicitly. Both methods are applied to the design of an airfoil for a hypothetical aircraft where the problem is formulated as an 18-point multipoint optimization.
\end{abstract}

\section{Introduction}

The aircraft design process applied in industry is a complex endeavour that involves concurrent engineering of the many systems that comprise a fully functional aircraft. In addition to the aerodynamic performance of an aircraft, equal consideration must be given to the disciplines tasked with specifying appropriate structures, controls, materials, and propulsion systems necessary to satisfy a truly comprehensive set of design requirements. In this day and age where greenhouse gas emissions associated with commercial aviation are of public concern with regard to climate change, and rising jet fuel prices are negatively impacting profits of commercial carriers, the design objective of improving aircraft fuel efficiency has become increasingly important. If it can be shown that a novel aerodynamic shape will provide the greatest improvement in aircraft fuel efficiency, then this shape should drive the design process such that the above mentioned disciplines strive to adapt their systems in order to minimize divergence from this optimal shape.

The coupling of computational fluid dynamics with numerical optimization techniques has resulted in aerodynamic shape optimization algorithms that are efficient at producing aircraft shape configurations with improved performance characteristics at a given aircraft operating condition. While significant progress in the field of aerodynamic shape optimization has been made over the past 20 years, further advancement is still required to make numerical optimization techniques useful to solve practical aerodynamic design problems. Practical aerodynamic design problems are characterized by design requirements that must be satisfied over a broad range of aircraft operating conditions. For aerodynamic shape optimization to be considered a viable alternative to the traditional cut-and-try approach to aerodynamic design, it must be capable of producing an optimal design that satisfies the design requirements over this broad range of

\footnotetext{
*Graduate Student, howard@oddjob.utias.utoronto.ca

${ }_{\dagger}^{\dagger}$ Undergraduate Student, Student Member AIAA, beckett@oddjob.utias.utoronto.ca

$\ddagger$ Professor, Tier I Canada Research Chair in Computational Aerodynamics, Associate Fellow AIAA, http://goldfinger.utias.utoronto.ca/ dwz
} 
operating conditions. This type of optimization, in which more than one aircraft operating condition is considered, is commonly referred to as multipoint optimization. Researchers have addressed the topic of multipoint optimization in various contexts within the realm of aerodynamic design problems. Recently, Epstein et al. ${ }^{3}$ used multipoint optimization to minimize wing drag at the main cruise operating condition and nearby secondary cruise operating conditions. Cliff et al. ${ }^{2}$ compare two approaches to multipoint optimization as applied to the aerodynamic shape optimization of the NASA Technology Concept Airplane, simultaneous multipoint design vs. sequential cruise-point design followed by trim optimization at transonic conditions. Zingg and Elias ${ }^{14}$ as well as Li, Huyse, and Padula ${ }^{5}$ have used multipoint optimization techniques applied to airfoil design to achieve constant drag over a range of cruise Mach numbers. Although significant contributions have been made by these researchers and others in the area of multipoint optimization, the scope of its application to aerodynamic design problems has been limited.

It is now appropriate that we define the terms on-design and off-design as they apply to the practical aerodynamic design problem presented in this paper. In our multipoint optimization problem formulation, on-design points refer to the operating conditions where we wish to optimize aerodynamic performance according to specified design objectives. For example, we may wish to minimize drag over a range of expected cruise Mach numbers and lift requirements. Our off-design points refer to operating conditions that can be considered aerodynamic constraints to the optimization. For example, the off-design requirement that an aerodynamic shape must be able to achieve a specified maximum lift coefficient at low speeds constrains the potential for drag minimization at cruise conditions. Note that although the off-design requirements are typically inequality constraints there is no benefit to surpassing the specifications.

The work described in this paper follows from the investigation of multipoint optimization applied to practical aerodynamic design problems undertaken by Zingg and Billing. ${ }^{13}$ Their goal was to demonstrate that multipoint optimization techniques can be applied to complex aerodynamic design problems that encompass a broad range of requirements extending beyond typical drag minimization over a range of cruise conditions. This broad range of design requirements includes high lift at low speed and consideration of maneuverability under dive conditions. The current work in progress focuses on several key findings from their investigation:

1. Performance at on-design points is compromised by the need to satisfy off-design constraints

2. On-design performance may be unnecessarily sacrificed if off-design constraints are over-satisfied.

3. Over-satisfaction of off-design constraints can be prevented by appropriate selection of their respective design point weights.

The design point weights mentioned above refer to the weights applied to the respective objective functions in the composite objective function. A problem with multipoint optimization noted by several researchers ${ }^{5,14}$ is that the appropriate off-design weights are not known a priori. As implied by the findings of Zingg and Billing, a poor assignment of off-design weights will result in one of two outcomes:

1. The off-design constraints are violated, or

2. The on-design performance is unnecessarily compromised

One might assume that there is an ideal weight value for any given off-design point that will result in a final optimized shape where its constraint value is exactly satisfied. However, a practical aerodynamic design problem may include off-design points that will have their constraints satisfied regardless of the weight applied to them, referred to as redundant points. Given this property of redundant off-design points, their ideal weight is zero.

This paper investigates the application of two different methods to solve a practical aerodynamic design problem. The goal of the first method using an unconstrained optimization algorithm is to determine the ideal weights for all of the off-design points considered in a practical aerodynamic design problem in a way that does not require user intervention. We introduce a procedure for automatically obtaining the ideal offdesign weights by exploiting aerodynamic performance trends as they evolve throughout the optimization. The second method uses the constrained optimization algorithm SNOPT, which allows the aerodynamic constraints imposed at the off-design operating conditions to be treated explicitly. The off-design points in this method are not included in the composite objective function (which contains only on-design objectives). 
Rather they are used as constraints in conjunction with the composite objective function to define a Lagrangian function that we seek to minimize to find the optimal solution. The following sections characterize, evaluate, and compare these two different approaches to solving practical aerodynamic design problems.

\section{Overview of Optima2D}

Both methods make use of the following tools contained within the computational code Optima2D:

- Two-dimensional turbulent flow solver

- Airfoil geometry parametrization

- Discrete adjoint gradient calculation

- Mesh movement algorithm

The compressible Reynolds-averaged Navier-Stokes equations are solved at each design iteration with the Newton-Krylov method developed by Nemec and Zingg7,8 in which the linear system arising at each Newton iteration is solved using the generalized minimal residual method (GMRES) preconditioned with an incomplete lower-upper (ILU) factorization with limited fill. Spatial derivatives in the governing equations are discretized using second-order centered finite differences with added scalar numerical dissipation. Eddy viscosity is computed using the one-equation Spalart-Allmaras turbulence model. The airfoil geometry is parametrized using B-spline control points. The vertical coordinates of these control points are considered design variables, thus allowing alterations to the baseline shape. For lift-constrained drag minimization problems, the design variables are B-spline control points, and the angle of attack is computed as part of the flow solution in order to meet the lift constraint (See Zingg and Billing ${ }^{13}$ for a description of this treatment of the lift constraint). For lift maximization problems, the angle of attack is a design variable in addition to the B-spline control points. Gradients of objective and constraint functions that are dependent on the flow solution are calculated using the discrete-adjoint method; the adjoint equation is solved using preconditioned GMRES. The first method uses an unconstrained quasi-Newton optimizer in which an estimate of the inverse Hessian based on the BFGS ( Broyden-Fletcher-Goldfarb-Shanno) rank-two update formula is used to compute a search direction. ${ }^{9}$ The step size is determined using a line search, which enforces the strong Wolfe conditions. The search direction and step size together, determine the new shape of the airfoil (and a new angle of attack in the case of lift maximization). Geometric constraints are added to the objective function as penalty terms. The second method uses the constrained optimization algorithm SNOPT, which is briefly described in Section VI. At each design iteration, the grid around the updated airfoil shape is perturbed using a simple algebraic grid movement technique.

\section{Design Problem Definition}

In order to define a practical multipoint optimization problem, we consider a hypothetical aircraft with a maximum weight of 100,000 lbs., a wing area of 1000 square feet, with a 35-degree sweep. The maximum cruise Mach number of the aircraft is 0.88 . We consider the section at the mean aerodynamic chord and assume that the sectional lift coefficient is equal to the wing lift coefficient. The target thickness to chord ratio is 0.118 . Regions of the flight envelope considered in this multipoint optimization include cruise, long-range cruise, dive, and low-speed conditions.

The first four operating conditions, labeled A-D in Table 1, correspond to cruise. Due to the sweep angle, the effective Mach number is 0.72. Two sets of operating weights and altitudes are considered. For operating point $\mathrm{A}$ the altitude is 29,000 feet, the weight is 60,000 lbs.; for $\mathrm{B}$ the altitude is the same, but the weight is 100,000 lbs.; for $\mathrm{C}$ the altitude is 39,000 feet, the weight is $60,000 \mathrm{lbs}$.; for $\mathrm{D}$ the altitude is 39,000 feet, the weight is $100,000 \mathrm{lbs}$. This leads to the Reynolds numbers and lift coefficients given in Table 1.

The next four operating conditions, labeled E-H, correspond to long-range cruise. The Mach number is 0.78 , producing an effective Mach number of 0.64 . The altitudes and weights are the same as for A-D respectively.

The objective for the eight operating points presented above is to minimize drag while maintaining their specified lift coefficients. These eight points represent the normal range of flight conditions under which the aircraft is designed to operate in the majority of its flight. Hence they are regarded as on-design points in 


\begin{tabular}{|c|c|c|c|c|}
\hline Operating Point & Reynolds Number & Mach Number & Lift Coefficient & $\mathcal{J}$ \\
\hline $\mathrm{A}$ & $27.32 \times 10^{6}$ & 0.72 & 0.17 & \multirow{8}{*}{$\frac{C_{d}}{C_{d}^{\circ}}$} \\
\hline $\mathrm{B}$ & $27.32 \times 10^{6}$ & 0.72 & 0.28 & \\
\hline $\mathrm{C}$ & $18.57 \times 10^{6}$ & 0.72 & 0.27 & \\
\hline $\mathrm{D}$ & $18.57 \times 10^{6}$ & 0.72 & 0.45 & \\
\hline $\mathrm{E}$ & $24.22 \times 10^{6}$ & 0.64 & 0.21 & \\
\hline $\mathrm{F}$ & $24.22 \times 10^{6}$ & 0.64 & 0.36 & \\
\hline G & $16.46 \times 10^{6}$ & 0.64 & 0.34 & \\
\hline $\mathrm{H}$ & $16.46 \times 10^{6}$ & 0.64 & 0.57 & \\
\hline $\mathrm{I}$ & $28.88 \times 10^{6}$ & 0.76 & 0.28 & \multirow{8}{*}{$\begin{array}{c}\frac{C_{d}}{C_{d}^{\circ}} \\
\left(M_{\max }<1.35\right)\end{array}$} \\
\hline $\mathrm{J}$ & $28.88 \times 10^{6}$ & 0.76 & 0.15 & \\
\hline $\mathrm{K}$ & $28.88 \times 10^{6}$ & 0.76 & 0.46 & \\
\hline $\mathrm{L}$ & $28.88 \times 10^{6}$ & 0.76 & 0.25 & \\
\hline M & $19.62 \times 10^{6}$ & 0.76 & 0.45 & \\
\hline $\mathrm{N}$ & $19.62 \times 10^{6}$ & 0.76 & 0.24 & \\
\hline $\mathrm{O}$ & $19.62 \times 10^{6}$ & 0.76 & 0.74 & \\
\hline $\mathrm{P}$ & $19.62 \times 10^{6}$ & 0.76 & 0.40 & \\
\hline Q & $11.8 \times 10^{6}$ & 0.16 & - & $\left(1-\frac{C_{l, \max }}{\widehat{C}_{l, \max }^{*}}\right)^{2}$ \\
\hline $\mathrm{R}$ & $15.0 \times 10^{6}$ & 0.20 & - & $\left(C_{l, \max }>1.60\right)$ \\
\hline
\end{tabular}

Table 1. Operating conditions for an 18-point optimization

this study. A complete problem specification could involve a careful prioritization by the designer of these operating conditions based on the knowledge of the aircraft mission requirements. In this study, we assume all on-design points are of equal importance.

The next eight operating conditions (I-P) are associated with the requirement for maneuverability under dive conditions. The flight Mach number is 0.93, making the effective Mach number 0.76. On top of the two sets of weights and altitudes considered as in the on-design points, two load factors are also taken into account. The combination produces a total of eight operating conditions. For operating point I, the altitude is $29,000 \mathrm{ft}$., the weight $60,000 \mathrm{lbs}$, and the load factor is 1.3 . For operating point J, the altitude and weight are the same, but the load factor is 0.7. Operating points $\mathrm{K}$ and L have an altitude of 29,000 ft., a weight of 100,000 lbs., and load factors 1.3 and 0.7, respectively. For operating points $\mathrm{M}$ and $\mathrm{N}$, the altitude is $39,000 \mathrm{ft}$., the weight is $60,000 \mathrm{lbs}$, and the load factors are 1.3 and 0.7 , respectively. Operating points $\mathrm{O}$ and $\mathrm{P}$ have the same altitude and load factors, but the weight is $100,000 \mathrm{lbs}$. The objective is to keep shock strengths modest, such that the upstream Mach number at all shocks is less than 1.35. The actual objective function used is again lift-constrained drag minimization, since this tends to weaken or eliminate shocks.

The final two operating points require an adequate maximum lift coefficient at low speed conditions. For operating condition Q, the altitude is sea level, the weight is 60,000 lbs., and the effective Mach number is 0.16 . For operating point $\mathrm{R}$ the weight is $100,000 \mathrm{lbs}$., and the effective Mach number is 0.20 . The objective is to ensure that the maximum attainable lift coefficient under these conditions is at least $1.75^{\mathrm{a}}$.

The last ten points, I-R, are considered off-design operating points. They impose constraints on the optimization. It is beneficial for the on-design performance if the off-design constraints are met, but not exceeded. These eighteen operating points span the flight envelope. This design problem definition is meant to illustrate a basic set of on-design and off-design specifications that can be used to formulate a multipoint optimization problem. In practice, additional operating conditions, such as climb, also need to be considered, but can be easily accommodated by the methods presented in this paper.

\section{Interpretation of Design Point Weights}

There are two terms that are frequently seen in the optimization literature, importance (or priority, of a design point) and difficulty (to improve a design point). Often these two terms are used interchangeably due to their mutual associations with optimization weights. If a design point is considered more important than

\footnotetext{
aThe optimization procedure applied to this design problem is demonstrated on a coarse mesh. Prior experience has shown that using a lower target lift coefficient of 1.60 on our coarse mesh will yield a lift coefficient of at least 1.75 on a finer mesh.
} 
others, higher weights will be assigned to it. Similarly, a common response to handling a design point that is difficult to improve is to increase its weight, creating the illusion that important points and difficult points should be treated in the same way. However, this multipoint optimization study reveals that it is crucial to distinguish between the two terms and clearly understand their respective influences since the philosophy behind each optimization weight adjustment strategy is shaped by the understanding of these two terms.

Consider the case of a two-point optimization starting at equal weights based on the two points having equal importance and having no prior knowledge of their respective difficulty. After the optimization converges for this hypothetical case, the drag of the first point is reduced by $50 \%$ while the drag of the second point is reduced by only $5 \%$. For the next optimization stage, presumably to allow further improvement, one may be tempted to increase the weight on the second point. By doing so, however, the optimization becomes implicitly driven by the difficulty factor. When a point is already in the vicinity of the physical limit to which it can be optimized, the reduction of the objective function will be small. Concentrating on the more difficult point, we forfeit the opportunity to achieve larger overall weighted objective function reduction obtainable by placing equal emphasis on both points based on their having equal importance. The path of the optimization is characterized by sets of optimization weights dictated by the difficulty factor. However this might not be in agreement with the importance factor of each design point. Hence adjusting on-design weights during the optimization based on difficulty is not logical without advanced knowledge of the priorities for the on-design points.

For the off-design points representing aerodynamic constraints the two factors work in harmony. An off-design point whose aerodynamic constraint is the most difficult to improve will be given more weight, implying that it is more important than other off-design points. In this case it is true, since this point is the bottleneck with respect to satisfying the off-design aerodynamic constraints.

\section{A Strategy for Obtaining Ideal Weights for Off-Design Points (Method 1)}

Practical aerodynamic design problems as defined in this paper are by nature constrained optimization problems. The goal of this general class of problems is to optimize performance at on-design operating conditions subject to aerodynamic constraints at off-design conditions. The off-design weight update strategy presented in this section employs an unconstrained BFGS optimization algorithm. In a sense, the weight update strategy attempts to emulate the behaviour of a constrained optimization algorithm. We have pursued this off-design weight update strategy because it allows us to take advantage of the strong legacy of work supporting our BFGS optimization algorithm.

\section{A. On-Design-First Optimization}

In this approach, we begin with a start-up optimization. The term on-design-first refers to this startup optimization, which includes only the on-design points. Performing an initial optimization with only on-design operating points serves two purposes. First, the initial airfoil may be poorly suited to the offdesign operating points, which may cause flow solver convergence difficulties. Performing the on-design-first optimization typically provides a better starting shape for introducing the off-design points. Second, it gives a clear picture of the performance trade-offs associated with the off-design constraints. The resultant airfoil geometry from the on-design-first optimization minimizes a weighted sum of the objective functions at all ondesign points. Under the assumption that all of the on-design operating conditions are of equal importance, all on-design objective functions are weighted equally; however the weight assignment is ultimately at the discretion of the designer, who may choose to weight the on-design points differently according to design priorities. This on-design-first airfoil is the starting point for the main optimization procedure that includes both the on-design points and the off-design points.

\section{B. Weight Update Formula}

For any given off-design point, there are 3 possibilities for the value of its respective aerodynamic constraint:

1. Constraint is violated

2. Constraint is exactly satisfied

3. Constraint is over-satisfied 
For off-design points where the aerodynamic constraints are violated, a higher weight is required on these points to pull them into the feasible region of the design space. For off-design points where the aerodynamic constraints are exactly satisfied, the weight is appropriate and does not require modification. For off-design points where constraints are over-satisfied, a lower weight is required to allow them to drift toward the boundary of the feasible region of the design space. As there is no particular benefit to over-satisfying an off-design aerodynamic constraint, it is desirable to shed weight on the off-design points that are in this category to reduce the negative impact on on-design performance. To facilitate the modification of off-design weights, a simple weight update formula is employed.

The concept for this approach to off-design weight modification can be credited to the work of Zingg and Elias $^{13}$ that demonstrates a similar technique for obtaining equal drag coefficients across a range of cruise Mach numbers by altering the objective function weights of design points in a multipoint optimization. For an aerodynamic constraint given by $\psi \leq \psi^{*}$, where $\psi$ is some functional, the weight update formula used in our procedure is:

$$
\omega_{n+1}=\omega_{n}\left(\frac{\psi_{n}}{\psi^{*}}\right)^{h}
$$

where $\psi_{n}$ is the current off-design aerodynamic constraint value, $\omega_{n}$ and $\omega_{n+1}$ are the current and updated off-design point weights, the exponent $h$ is a user defined parameter that affects the magnitude of the weight change, and $n$ is the index of weight update cycles.

For off-design points representing requirements for $C_{l, \max }$, the values of $\psi_{n}$ and $\psi^{*}$ to be used in the weight update formula are:

$$
\begin{aligned}
& \psi_{n}=\frac{1}{C_{l, \text { max }}^{n}} \\
& \psi^{*}=\frac{1}{C_{l, \text { max }}^{*}}
\end{aligned}
$$

For off-design points with a maximum local Mach number constraint, $M_{\max } \leq M_{\text {max }}^{*}$, the values of $\psi_{n}$ and $\psi^{*}$ to be used in the weight update formula are defined in equations 4 and 5 respectively.

$$
\begin{aligned}
& \psi_{n}=M_{\text {max }}^{n} \\
& \psi^{*}=M_{\text {max }}^{*}
\end{aligned}
$$

The first application of the weight update formula to obtain initial off-design weights $\omega_{1}$ requires special treatment because there are no previous weights to use in the formula. An arbitrary value of unity is assigned to $\omega_{0}$ for all off-design points. The off-design constraint values $\psi_{0}$ are evaluated using the on-design-first airfoil geometry. Initial off-design weights $\omega_{1}$ are then calculated using the weight update formula with $\omega_{0}=1$. It is important to clarify that the $\omega_{0}$ weights are not used at any time during the optimization procedure; they only facilitate the calculation of $\omega_{1}$. The weight update formula is subsequently used at regular intervals (after every weight update cycle) throughout the main optimization procedure to update the off-design point weights.

\section{Weight Update Cycles}

The weights of the off-design points are updated periodically based on the values of their respective aerodynamic constraints during the course of the optimization. A weight update cycle consists of a user-specified number of optimization iterations followed by an evaluation of the off-design aerodynamic constraints and corresponding update of the off-design weights. A new weight update cycle begins with a restarted optimization using the updated off-design weights and the final airfoil geometry from the previous weight update cycle. If the magnitude of the change in the updated weights is less than a user-specified tolerance, the optimization leaves the weights unchanged and continues until the next weight update cycle. In this manner, weight update cycles are executed until a converged optimal solution is obtained. At the converged optimal solution, the off-design weights are as low as possible while satisfying all of the off-design aerodynamic constraints. 


\section{An Alternative Strategy Using Constrained Optimization (Method 2)}

An alternative strategy for solving practical aerodynamic design problems utilizes the SNOPT algorithm for constrained optimization problems developed by Gill, Murray and Saunders. ${ }^{4}$ The SNOPT algorithm allows us to treat the off-design operating conditions as explicit constraints within the framework of a constrained optimization problem. SNOPT uses a sequential quadratic programming (SQP) method that obtains search directions from a sequence of quadratic programming (QP) subproblems. Each QP subproblem minimizes a quadratic model of a Lagrangian function which is used to represent an objective function subject to linearized constraints.

\section{A. On-Design-First Optimization with SNOPT}

As with the off-design weight update strategy, an on-design-first optimization is performed to obtain a favourable airfoil geometry for use as a starting point before the introduction of the off-design constraints. SNOPT is used to minimize a weighted sum of the objective functions at all on-design points. This composite objective function is subject only to geometric thickness constraints which are satisfied explicitly by SNOPT.

\section{B. KS Function Used for Evaluation of Maximum Mach Number Constraints}

Off-design points I-P representing dive conditions are subject to the constraint that the maximum Mach number in the flow field not exceed 1.35. The maximum Mach number function used to represent this constraint is not continuous with respect to the design variables and therefore cannot be handled directly by SNOPT. Since the Mach number at each node is continuous with respect to the design variables, we could assign our Mach number constraint to each node in the flow field. However, performing an adjoint gradient evaluation of the Mach number constraint at each node would be prohibitively expensive. Instead we use the Kreisselmeier-Steinhauser $(K S)$ function as a means to aggregate the Mach number constraints at all nodes in the flow field into a single composite function that is continuously differentiable. The $K S$ function produces an envelope surface that is $C_{1}$ continuous and represents a conservative estimate of the maximum among the set of constraint functions considered. ${ }^{12}$ The $K S$ function has been used in various applications where constraint aggregation is required for efficient use of gradient-based optimization methods including aerodynamic shape optimization ${ }^{1}$ and aircraft design. ${ }^{6,11}$ An alternate formulation of the $K S$ function proposed by Poon and Martins ${ }^{10}$ is used to reduce numerical difficulties associated with the original formulation and is given by:

$$
K S[\mathbf{g}(\mathbf{x})]=g_{\max }(\mathbf{x})+\frac{1}{\rho} \ln \left[\sum_{j=1}^{m} e^{\rho\left[g_{j}(x)-g_{\max }(x)\right]}\right]
$$

where $\mathbf{g}(\mathbf{x})$ contains the set of constraints, $g_{\max }$ is the maximum constraint value evaluated at the current design iteration, $\mathbf{x}$, and $\rho$ is the draw-down parameter that governs the conservativeness of the estimate of $g_{\max }$ such that

$$
\lim _{\rho \rightarrow \infty} K S(\mathbf{x}, \rho)=g_{\max }(\mathbf{x})
$$

The constraints $g_{j}(\mathbf{x})$ are evaluated at every node in the flow field with the exception of the nodes on the surface of the airfoil and are given by:

$$
g_{j}(\mathbf{x})=\frac{M_{j}(\mathbf{x})}{M^{*}}-1
$$

where $M^{*}=1.35$ is the upper bound on the Mach number constraint, $M_{j}(\mathbf{x})$ is the Mach number at node $j$ at the current design iteration $\mathbf{x}$ and the constraint is considered satisfied if $g_{j}(\mathbf{x}) \leq 0 . g_{\max }$ is given by:

$$
g_{\max }(\mathbf{x})=\frac{M_{\max }(\mathbf{x})}{M^{*}}-1
$$

where $M_{\max }(\mathbf{x})$ is the maximum Mach number in the flow field at the current design iteration $\mathbf{x}$.

A conservative estimate of the maximum Mach number is given by:

$$
M_{k s}(K S)=(K S+1) M^{*}
$$


The actual constraint that SNOPT works with is $M_{k s}$. To obtain a solution that satisfies the desired maximum Mach number constraint, $M^{*}$, appropriate values of $\rho$ and the $M_{k s}$ target must be specified and are case dependent. Currently, an ad-hoc approach is taken to determine these values.

\section{Evaluation of High-Lift Constraints}

Off-design points Q-R representing design requirements for high lift at low speeds are subject to the constraint that $C_{l, \max }$ must be at least 1.60. The constraint function assigned to these points is given simply as $C_{l}$.

\section{Off-Design Constraint Function Gradients}

The gradients of the constraint functions $M_{k s}$ and $C_{l}$ with respect to the design variables, $\mathbf{x}$, are computed using the discrete adjoint approach.

\section{Results}

\section{A. Multipoint Optimization Setup}

For all cases, the airfoil geometry is parametrized using 15 B-spline control points. Three control points are frozen at the leading edge and two at the trailing edge. The remaining 10 control points are designated as design variables and are split evenly between the top and bottom airfoil surfaces. A floating thickness constraint of $11.9 \%$ chord is imposed to ensure a thickness of at least $11.8 \%$ chord. In addition, thickness constraints of $1 \%$ chord and $0.2 \%$ chord are imposed at $95 \%$ chord and $99 \%$ chord respectively to prevent trailing edge crossover. The latter is typically inactive once convergence is achieved. The meshes used have a C topology with 289 nodes in the streamwise direction and 65 nodes in the normal direction; the off-wall spacing is $10^{-6}$ chord. Cases 1 to 3 use the off-design weight update method described in Section V. Cases 4 and 5 use the constrained optimization method described in Section VI.

\section{Off-Design Weight Update Setup For Cases 1 - 3}

For design points A through $\mathrm{P}$, the design objective is lift-constrained drag minimization. For each of these design points, the corresponding objective function is the drag coefficient normalized by the drag coefficient evaluated using the initial airfoil geometry. For design points $\mathrm{Q}$ and $\mathrm{R}$, the design objective is to meet minimum $C_{l, \max }$ requirements needed at low speeds. For these two design points, the corresponding objective function is given by:

$$
J=\left(1-\frac{C_{l, \max }}{\widehat{C}_{l, \text { max }}^{*}}\right)^{2}
$$

where $\widehat{C}_{l, \text { max }}^{*}$ is a target maximum lift coefficient specific to the objective function not to be confused with the desired target maximum lift coefficient $C_{l, \max }^{*}$ (see equation 3) used in the weight update formula. The value of $\widehat{C}_{l, \text { max }}^{*}$ is set to a value that is unattainable and hence this corresponds to lift maximization. The optimization algorithm will strive to attain the objective function-specific target lift value $\widehat{C}_{l, \max }^{*}$ at points $\mathrm{Q}$ and $\mathrm{R}$ while their corresponding weights will be adjusted to ensure that the desired target lift value $C_{l, \max }^{*}$ is obtained. Also for points $\mathrm{Q}$ and $\mathrm{R}$, the angle of attack is included among the design variables. This objective function is again normalized by its initial value.

A composite objective function is formed by a weighted sum of the individual objective functions and is given by:

$$
J=\omega_{A} J_{A}+\omega_{B} J_{B}+\ldots+\omega_{R} J_{R}
$$

where the subscripts indicate the operating points, and the $\omega$ s are the weights. The weights for the on-design points A - H remain fixed at unity throughout the duration of the optimization procedure. The weights for the off-design points I - $\mathrm{R}$ are periodically modified throughout the optimization procedure according to the strategy described in Section V. Ten optimization iterations are executed per weight update cycle. The value of the exponent used in the weight update formula, equation (1), is $h=4$. 


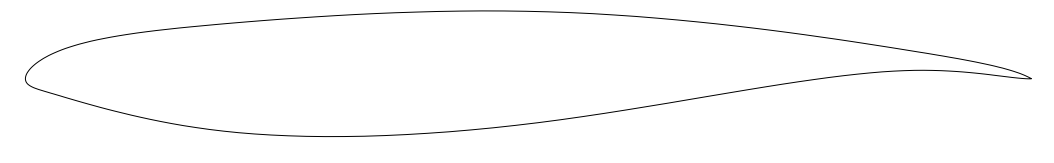

Figure 1. Case 1 - Optimized airfoil after 33 weight update cycles

\section{Constrained Optimization Setup For Cases 4 - 5}

For on-design points A-H, the design objective is lift-constrained drag minimization. For each of these design points, the corresponding objective function is the drag coefficient normalized by the drag coefficient evaluated using the initial airfoil geometry. A composite objective function is formed by a weighted sum of the individual on-design objective functions with all weights equal to unity. The off-design constraints are defined as described in Section VI. A value of $\rho=31$ is used in the $K S$ function. The target value of $M_{k s}$ used by SNOPT is 1.50 . This produces $M_{\max } \leq 1.35$ as desired.

\section{B. Case 1 - Baseline 18 Point Optimization}

The initial geometry used for the optimization in this case is the RAE 2822 airfoil. The full set of 18 design points, A-R, described in Table 1 are used.

The results for Case 1 presented here were obtained after 33 weight update cycles. The optimization was terminated at 33 weight update cycles when it was observed that the change in on-design drag values from cycle to cycle was significantly less than the numerical error in drag values expected from the coarse mesh used in this study. The optimized airfoil obtained is shown in Figure 1. Tables 2, 3, and 4 provide a summary of the on-design and off-design performance values for the initial RAE 2822 airfoil, the on-designfirst airfoil, and the airfoil after 33 weight update cycles. For the on-design-first airfoil and the optimized airfoil after 33 weight update cycles, the tabulated performance results were obtained from regenerated meshes with the same properties as the original mesh for greater accuracy ${ }^{\mathrm{b}}$. Note that for the RAE 2822 airfoil the off-design constraints at points $\mathrm{O}, \mathrm{Q}$, and $\mathrm{R}$ are violated. After the on-design-first optimization, the off-design constraints at points $\mathrm{O}, \mathrm{Q}$, and $\mathrm{R}$, are still violated. The final optimized airfoil for Case 1 has roughly maintained the drag coefficient values at the eight on-design points compared to the initial RAE 2822 airfoil while satisfying all off-design constraints. A comparison of the drag coefficients at the on-design points for the final optimized airfoil versus the on-design-first airfoil show that there has been degradation in performance, but the severity of the degradation has been mitigated by use of the automated weight update procedure. Pressure distributions for all on-design points A-H are shown in Figures 10 and 11. The solutions are shock free at all eight on-design operating conditions.

\section{Off-Design Points}

Figures 2 and 3 show the evolution of the constraint values for off-design points I - P and Q and R, respectively, throughout the optimization procedure. Figure 4 shows corresponding off-design weights. At the beginning of the main optimization procedure (after the start-up on-design-first optimization but before the first weight update cycle), the off-design aerodynamic constraints are violated at points $O, Q$, and R, while the remaining off-design aerodynamic constraints are satisfied. Since some off-design aerodynamic constraints are not satisfied, we must proceed with the weight update cycles, which will result in degradation in on-design performance. The off-design weights begin at their initial reference values of unity. Within the first several weight update cycles, two distinct categories of off-design points emerge from these plots:

1. Active off-design points

2. Redundant off-design points

\footnotetext{
${ }^{\mathrm{b}}$ During the course of an optimization if the shape of the airfoil has changed significantly from the initial geometry, degradation in the quality of the mesh is caused by inherent limitations of the algebraic mesh movement algorithm. The degradation in mesh quality leads to increased error in the flow solution and corresponding lift and drag values.
} 


\begin{tabular}{|c|c|c|c|c|c|}
\hline Operating Point & $\alpha$ & $C_{l}$ & $C_{d}$ & $M_{\max }$ & $C_{l, \max }$ \\
\hline \hline $\mathrm{A}$ & $-0.71^{\circ}$ & 0.17 & 0.0090 & - & - \\
$\mathrm{B}$ & $-0.06^{\circ}$ & 0.28 & 0.0092 & - & - \\
$\mathrm{C}$ & $-0.12^{\circ}$ & 0.27 & 0.0101 & - & - \\
$\mathrm{D}$ & $+0.93^{\circ}$ & 0.45 & 0.0108 & - & - \\
$\mathrm{E}$ & $-0.36^{\circ}$ & 0.21 & 0.0090 & - & - \\
$\mathrm{F}$ & $+0.66^{\circ}$ & 0.36 & 0.0094 & - & - \\
$\mathrm{G}$ & $+0.53^{\circ}$ & 0.34 & 0.0104 & - & - \\
$\mathrm{H}$ & $+2.10^{\circ}$ & 0.57 & 0.0115 & - & - \\
\hline \hline $\mathrm{I}$ & $-0.17^{\circ}$ & 0.28 & 0.0100 & 1.20 & - \\
$\mathrm{J}$ & $-0.85^{\circ}$ & 0.15 & 0.0092 & 1.10 & - \\
$\mathrm{K}$ & $+0.87^{\circ}$ & 0.46 & 0.0136 & 1.30 & - \\
$\mathrm{L}$ & $-0.33^{\circ}$ & 0.25 & 0.0097 & 1.17 & - \\
$\mathrm{M}$ & $+0.77^{\circ}$ & 0.45 & 0.0141 & 1.30 & - \\
$\mathrm{N}$ & $-0.39^{\circ}$ & 0.24 & 0.0105 & 1.16 & - \\
$\mathrm{O}$ & $+3.46^{\circ}$ & 0.74 & 0.0383 & 1.40 & - \\
$\mathrm{P}$ & $+0.48^{\circ}$ & 0.40 & 0.0128 & 1.26 & - \\
\hline \hline $\mathrm{Q}$ & $+16.50^{\circ}$ & - & - & - & 1.43 \\
$\mathrm{R}$ & $+16.50^{\circ}$ & - & - & - & 1.40 \\
\hline
\end{tabular}

Table 2. Baseline airfoil performance (RAE 2822)

\begin{tabular}{|c|c|c|c|c|c|}
\hline Operating Point & $\alpha$ & $C_{l}$ & $C_{d}$ & $M_{\max }$ & $C_{l, \max }$ \\
\hline \hline $\mathrm{A}$ & $-0.45^{\circ}$ & 0.17 & 0.0086 & - & - \\
$\mathrm{B}$ & $+0.20^{\circ}$ & 0.28 & 0.0088 & - & - \\
$\mathrm{C}$ & $+0.14^{\circ}$ & 0.27 & 0.0097 & - & - \\
$\mathrm{D}$ & $+1.19^{\circ}$ & 0.45 & 0.0105 & - & - \\
$\mathrm{E}$ & $-0.09^{\circ}$ & 0.21 & 0.0087 & - & - \\
$\mathrm{F}$ & $+0.94^{\circ}$ & 0.36 & 0.0092 & - & - \\
$\mathrm{G}$ & $+0.82^{\circ}$ & 0.34 & 0.0100 & - & - \\
$\mathrm{H}$ & $+2.40^{\circ}$ & 0.57 & 0.0112 & - & - \\
\hline \hline $\mathrm{I}$ & $+0.08^{\circ}$ & 0.28 & 0.0105 & 1.25 & - \\
$\mathrm{J}$ & $-0.62^{\circ}$ & 0.15 & 0.0096 & 1.21 & - \\
$\mathrm{K}$ & $+1.25^{\circ}$ & 0.46 & 0.0174 & 1.35 & - \\
$\mathrm{L}$ & $-0.09^{\circ}$ & 0.25 & 0.0100 & 1.23 & - \\
$\mathrm{M}$ & $+1.14^{\circ}$ & 0.45 & 0.0175 & 1.35 & - \\
$\mathrm{N}$ & $-0.14^{\circ}$ & 0.24 & 0.0106 & 1.22 & - \\
$\mathrm{O}$ & $+4.72^{\circ}$ & 0.74 & 0.0513 & 1.45 & - \\
$\mathrm{P}$ & $+0.80^{\circ}$ & 0.40 & 0.0150 & 1.33 & - \\
\hline \hline $\mathrm{Q}$ & $+11.40^{\circ}$ & - & - & - & 1.27 \\
$\mathrm{R}$ & $+10.80^{\circ}$ & - & - & - & 1.23 \\
\hline
\end{tabular}

Table 3. On-design-first airfoil performance (Case 1) 


\begin{tabular}{|c|c|c|c|c|c|}
\hline Operating Point & $\alpha$ & $C_{l}$ & $C_{d}$ & $M_{\max }$ & $C_{l, \max }$ \\
\hline \hline $\mathrm{A}$ & $-1.61^{\circ}$ & 0.17 & 0.0092 & - & - \\
$\mathrm{B}$ & $-0.97^{\circ}$ & 0.28 & 0.0093 & - & - \\
$\mathrm{C}$ & $-1.02^{\circ}$ & 0.27 & 0.0102 & - & - \\
$\mathrm{D}$ & $+0.02^{\circ}$ & 0.45 & 0.0107 & - & - \\
$\mathrm{E}$ & $-1.36^{\circ}$ & 0.21 & 0.0092 & - & - \\
$\mathrm{F}$ & $-0.34^{\circ}$ & 0.36 & 0.0095 & - & - \\
$\mathrm{G}$ & $-0.46^{\circ}$ & 0.34 & 0.0104 & - & - \\
$\mathrm{H}$ & $+1.11^{\circ}$ & 0.57 & 0.0113 & - & - \\
\hline \hline $\mathrm{I}$ & $-1.02^{\circ}$ & 0.28 & 0.0106 & 1.21 & - \\
$\mathrm{J}$ & $-1.66^{\circ}$ & 0.15 & 0.0120 & 1.32 & - \\
$\mathrm{K}$ & $-0.04^{\circ}$ & 0.46 & 0.0129 & 1.28 & - \\
$\mathrm{L}$ & $-1.17^{\circ}$ & 0.25 & 0.0107 & 1.24 & - \\
$\mathrm{M}$ & $-0.09^{\circ}$ & 0.45 & 0.0135 & 1.28 & - \\
$\mathrm{N}$ & $-1.21^{\circ}$ & 0.24 & 0.0115 & 1.25 & - \\
$\mathrm{O}$ & $+1.46^{\circ}$ & 0.74 & 0.0201 & 1.35 & - \\
$\mathrm{P}$ & $-0.37^{\circ}$ & 0.40 & 0.0125 & 1.25 & - \\
\hline \hline $\mathrm{Q}$ & $+14.64^{\circ}$ & - & - & - & 1.63 \\
$\mathrm{R}$ & $+14.47^{\circ}$ & - & - & - & 1.63 \\
\hline
\end{tabular}

Table 4. Airfoil performance after 33 weight update cycles (Case 1)

Points $\mathrm{O}, \mathrm{Q}$, and $\mathrm{R}$ can be considered active. These points require non-zero weightings to prevent violation of their target aerodynamic constraint values. It is clear from Figure 4 that the off-design constraint at point $\mathrm{O}$ is the most difficult to satisfy, as illustrated by its high weight compared to the other active points $\mathrm{Q}$, and R. Points J, I, K, L, M, N, and P can be considered redundant. These points will have their aerodynamic constraints satisfied regardless of the weight applied to them. Redundant points have their aerodynamic constraints satisfied by virtue of their proximity to active points with similar operating conditions. The weight update strategy recognizes this property of the redundant off-design points and accordingly reduces their weights to zero, as shown in Figure 4.

The behaviour of the active off-design points is characterized by an initial period of growth followed by asymptotic convergence of their aerodynamic constraints to their respective target values with corresponding constant weight values. The redundant points attain constant over-satisfied aerodynamic constraint values with corresponding weightings of zero. This behaviour is observed in Figures 2, 3, and 4.

It is likely that point $\mathrm{Q}$ is also redundant. Throughout the optimization procedure, the off-design constraint value at point $\mathrm{Q}, C_{l, \max }$, follows slightly above the $C_{l, \max }$ constraint value at point $\mathrm{R}$, as shown in Figure 3. It appears that as long as the $C_{l, \max }$ constraint at point $\mathrm{R}$ is satisfied, the $C_{l, \max }$ constraint at point $\mathrm{Q}$ will also be satisfied with a slightly higher value. The claim of redundancy of point $\mathrm{Q}$ can be further justified by noting that its weight is slowly approaching zero while the weight on point $\mathrm{R}$ remains constant during the last 10 weight update cycles. It is reasonable to assume that if the optimization were allowed to continue beyond 33 weight update cycles, the weight for point $\mathrm{Q}$ would eventually reach zero.

\section{On-Design Points}

Since the on-design optimization weights are held constant throughout the optimization procedure, the ondesign performance is evolving through the course of the optimization solely due to the shift of emphasis characterized by the relative change in the off-design weights. Figure 5 shows the evolution of the drag coefficients for the eight on-design points. In the first 7 weight update cycles, significant on-design performance fluctuation is clearly observable. With the introduction of the off-design points, the on-design drag values immediately begin to rise. Figure 6 shows the sum of the on-design drag coefficients superimposed against the sum of the off-design weights. It illustrates the relationship between on-design performance and off-design weights. 


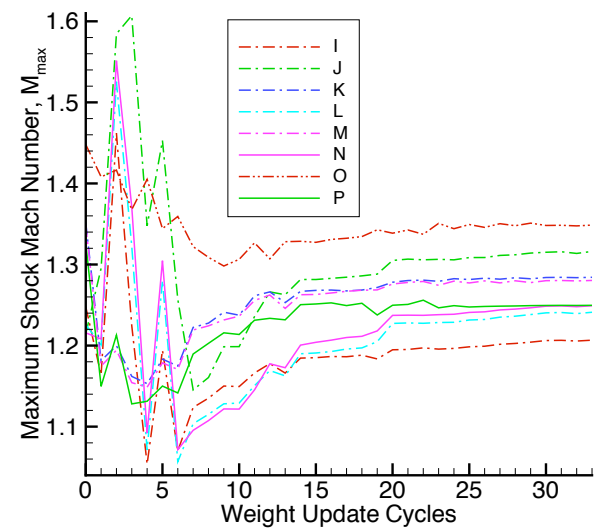

Figure 2. Evolution of off-design constraint values I - P

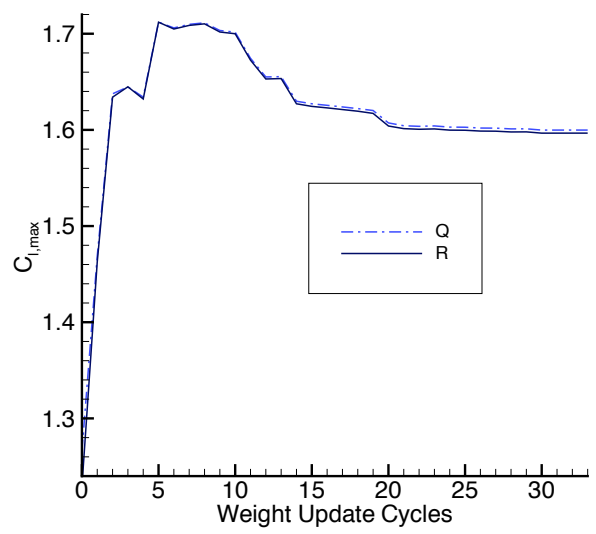

Figure 3. Evolution of off-design aerodynamic constraint values $Q$ and $\mathbf{R}$

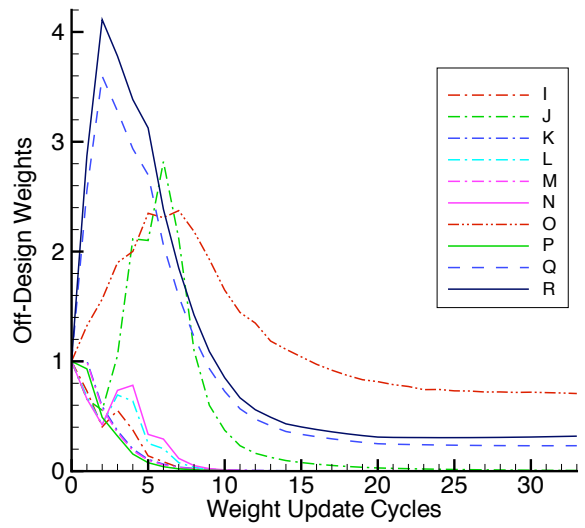

Figure 4. Evolution of off-design weights 


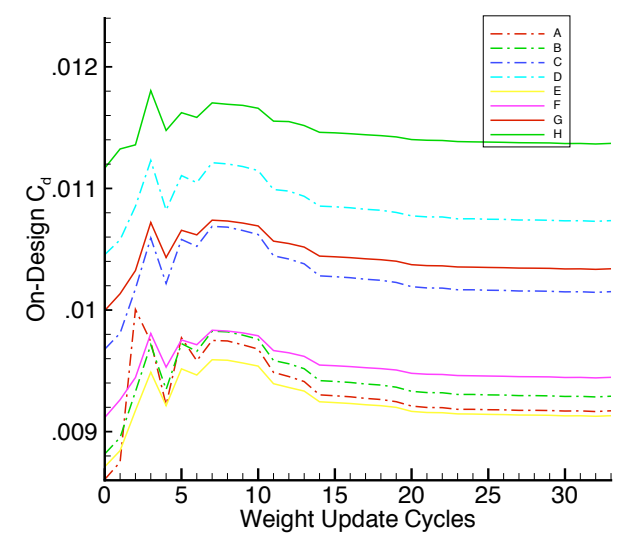

Figure 5. Evolution of on-design performance

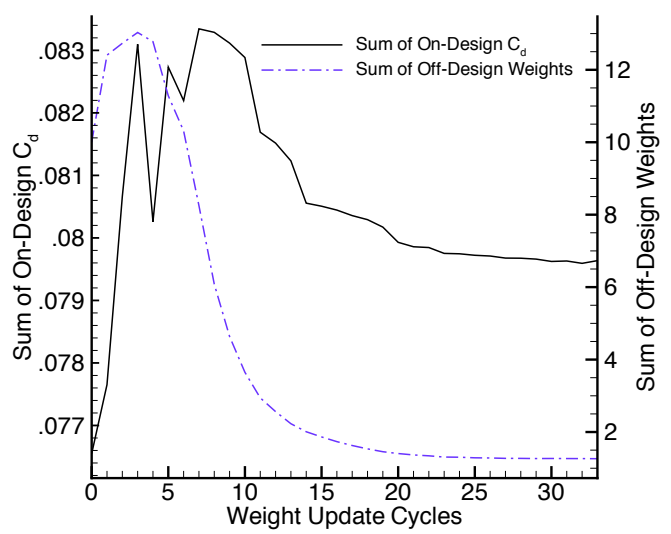

Figure 6. Sum of on-design performance values versus sum of off-design weights

\section{Case 2 - Alternate Initial Airfoil}

The initial geometry used for the optimization in this case is the NACA 0012 airfoil. The full set of 18 design points, A-R, described in Table 1 are used.

The results shown were obtained after 36 weight update cycles. A comparison of the optimized airfoil obtained in Case 2 versus the optimized airfoil obtained in Case 1 is shown in Figure 7 . Table 5 shows a comparison of the performance values for the final optimized airfoil for Case 2 versus the baseline NACA 0012 airfoil performance values. The slight difference in optimized airfoil geometries can be attributed to differences in the design spaces in each case resulting from the application of the mesh movement algorithm starting from two different initial airfoil geometries. The substantial improvement in performance for the final optimized airfoil compared to the baseline NACA 0012 airfoil performance indicates that the NACA 0012 airfoil is poorly suited to the design problem studied in this paper. In contrast, the RAE 2822 airfoil used in Case 1 is a more appropriate first guess as a solution to this design problem in terms of initial ondesign performance and violation of off-design constraints. These results show that the final airfoil geometry and performance values for Case 2 are very similar to results obtained for Case 1 . This is significant because it confirms that the automated weight update procedure will produce the same final optimized results independent of the initial geometry. 


\begin{tabular}{|c|c|c|c|c|c|c|c|c|c|c|}
\hline \multirow{2}{*}{ Operating Point } & \multicolumn{9}{|c|}{ NACA 0012 } & \multicolumn{5}{c|}{ Case 2 } \\
\cline { 2 - 10 } & $\alpha$ & $C_{l}$ & $C_{d}$ & $M_{\max }$ & $C_{l, \max }$ & $\alpha$ & $C_{l}$ & $C_{d}$ & $M_{\max }$ & $C_{l, \max }$ \\
\hline \hline $\mathrm{A}$ & $+1.01^{\circ}$ & 0.17 & 0.0092 & - & - & $-1.59^{\circ}$ & 0.17 & 0.0092 & - & - \\
$\mathrm{B}$ & $+1.67^{\circ}$ & 0.28 & 0.0105 & - & - & $-0.95^{\circ}$ & 0.28 & 0.0093 & - & - \\
$\mathrm{C}$ & $+1.61^{\circ}$ & 0.27 & 0.0111 & - & - & $-1.00^{\circ}$ & 0.27 & 0.0102 & - & - \\
$\mathrm{D}$ & $+2.82^{\circ}$ & 0.45 & 0.0188 & - & - & $+0.05^{\circ}$ & 0.45 & 0.0107 & - & - \\
$\mathrm{E}$ & $+1.45^{\circ}$ & 0.21 & 0.0093 & - & - & $-1.32^{\circ}$ & 0.21 & 0.0092 & - & - \\
$\mathrm{F}$ & $+2.48^{\circ}$ & 0.36 & 0.0100 & - & - & $-0.30^{\circ}$ & 0.36 & 0.0094 & - & - \\
$\mathrm{G}$ & $+2.35^{\circ}$ & 0.34 & 0.0108 & - & - & $-0.42^{\circ}$ & 0.34 & 0.0104 & - & - \\
$\mathrm{H}$ & $+3.95^{\circ}$ & 0.57 & 0.0136 & - & - & $+1.15^{\circ}$ & 0.57 & 0.0114 & - & - \\
\hline \hline $\mathrm{I}$ & $+1.57^{\circ}$ & 0.28 & 0.0160 & 1.34 & - & $-0.99^{\circ}$ & 0.28 & 0.0108 & 1.24 & - \\
$\mathrm{J}$ & $+0.81^{\circ}$ & 0.15 & 0.0110 & 1.27 & - & $-1.67^{\circ}$ & 0.15 & 0.0100 & 1.22 & - \\
$\mathrm{K}$ & $+3.06^{\circ}$ & 0.46 & 0.0337 & 1.47 & - & $-0.04^{\circ}$ & 0.46 & 0.0143 & 1.31 & - \\
$\mathrm{L}$ & $+1.38^{\circ}$ & 0.25 & 0.0146 & 1.32 & - & $-1.15^{\circ}$ & 0.25 & 0.0105 & 1.22 & - \\
$\mathrm{M}$ & $+2.92^{\circ}$ & 0.45 & 0.0326 & 1.46 & - & $-0.09^{\circ}$ & 0.45 & 0.0149 & 1.29 & - \\
$\mathrm{N}$ & $+1.33^{\circ}$ & 0.24 & 0.0149 & 1.32 & - & $-1.21^{\circ}$ & 0.24 & 0.0112 & 1.21 & - \\
$\mathrm{O}$ & $+15.50^{\circ}$ & 0.74 & 0.2545 & 1.68 & - & $+1.46^{\circ}$ & 0.74 & 0.0207 & 1.33 & - \\
$\mathrm{P}$ & $+2.42^{\circ}$ & 0.40 & 0.0261 & 1.42 & - & $-0.37^{\circ}$ & 0.40 & 0.0137 & 1.29 & - \\
\hline \hline $\mathrm{Q}$ & $+15.00^{\circ}$ & - & - & - & 1.45 & $+14.61^{\circ}$ & - & - & - & 1.64 \\
$\mathrm{R}$ & $+15.00^{\circ}$ & - & - & - & 1.44 & $+14.39^{\circ}$ & - & - & - & 1.64 \\
\hline
\end{tabular}

Table 5. Case 2 optimized airfoil performance versus baseline NACA 0012 airfoil performance

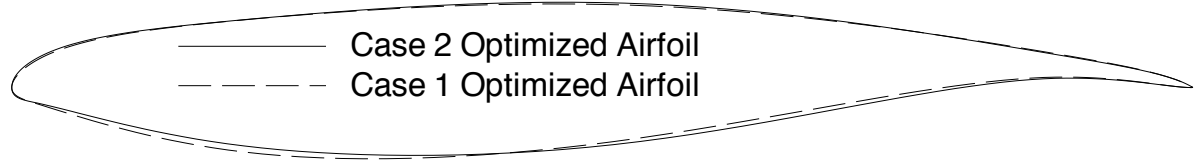

Figure 7. Case 2 optimized airfoil versus Case 1 optimized airfoil

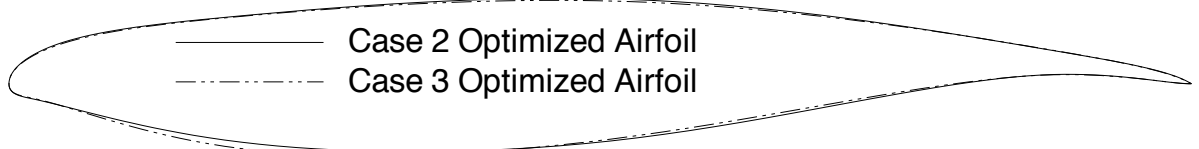

Figure 8. Case 3 optimized airfoil versus Case 2 optimized airfoil

\section{Case 3 - Reduced Set of Off-Design Points}

The initial geometry used for the optimization in this case is the NACA 0012 airfoil. A reduced set of design points is used for this case. On-design points A-H are included, but only off-design points $\mathrm{O}$ and $\mathrm{R}$ are included for a total of 10 design points. Points $\mathrm{O}$ and $\mathrm{R}$ are the only off-design points included because the results from Case 1 revealed that the other off-design points are redundant.

Table 6 shows the performance values for the optimized airfoil obtained after 33 weight update cycles and a comparison of the optimized airfoil geometries from Case 3 versus Case 2 are shown in Figure 8. These results show that the final airfoil geometry and performance values for Case 3 are very similar to results obtained for Case 2. In practice, the knowledge regarding the redundancy of the off-design points would not be available prior to running the optimization. However it is instructive to show that the same 


\begin{tabular}{|c|c|c|c|c|c|}
\hline Operating Point & $\alpha$ & $C_{l}$ & $C_{d}$ & $M_{\max }$ & $C_{l, \max }$ \\
\hline \hline $\mathrm{A}$ & $-1.55^{\circ}$ & 0.17 & 0.0092 & - & - \\
$\mathrm{B}$ & $-0.91^{\circ}$ & 0.28 & 0.0093 & - & - \\
$\mathrm{C}$ & $-0.96^{\circ}$ & 0.27 & 0.0102 & - & - \\
$\mathrm{D}$ & $+0.09^{\circ}$ & 0.45 & 0.0107 & - & - \\
$\mathrm{E}$ & $-1.29^{\circ}$ & 0.21 & 0.0092 & - & - \\
$\mathrm{F}$ & $-0.27^{\circ}$ & 0.36 & 0.0094 & - & - \\
$\mathrm{G}$ & $-0.39^{\circ}$ & 0.34 & 0.0104 & - & - \\
$\mathrm{H}$ & $+1.18^{\circ}$ & 0.57 & 0.0113 & - & - \\
\hline \hline $\mathrm{I}$ & $-0.95^{\circ}$ & 0.28 & 0.0108 & 1.22 & - \\
$\mathrm{J}$ & $-1.60^{\circ}$ & 0.15 & 0.0123 & 1.33 & - \\
$\mathrm{K}$ & $-0.03^{\circ}$ & 0.46 & 0.0130 & 1.28 & - \\
$\mathrm{L}$ & $-1.10^{\circ}$ & 0.25 & 0.0108 & 1.25 & - \\
$\mathrm{M}$ & $-0.02^{\circ}$ & 0.45 & 0.0136 & 1.28 & - \\
$\mathrm{N}$ & $-1.14^{\circ}$ & 0.24 & 0.0117 & 1.26 & - \\
$\mathrm{O}$ & $+1.51^{\circ}$ & 0.74 & 0.0198 & 1.34 & - \\
$\mathrm{P}$ & $-0.30^{\circ}$ & 0.40 & 0.0125 & 1.25 & - \\
\hline \hline $\mathrm{Q}$ & $+14.61^{\circ}$ & - & - & - & 1.63 \\
$\mathrm{R}$ & $+14.56^{\circ}$ & - & - & - & 1.62 \\
\hline
\end{tabular}

Table 6. Case 3 optimized airfoil performance

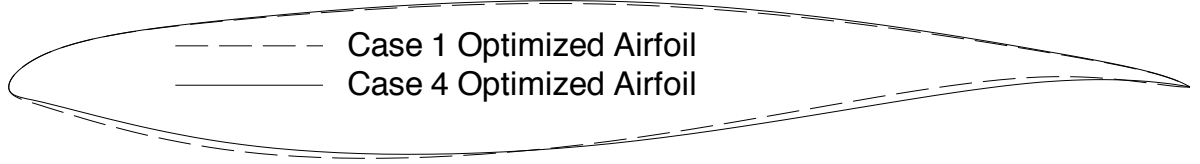

Figure 9. Case 4 - Optimized airfoil after 44 SNOPT major iterations

optimized results are obtained with significantly less computational effort. Both cases required a similar number of design iterations and weight update cycles, but only 10 flow solves and adjoint calculations per design iteration were required for this case compared to 18 for Case 2. A logical extension of this work would be to investigate methods to predict redundancy of off-design points, although an experienced designer may be able to do so successfully.

\section{E. Case 4 - Full Set of Off-Design Points Using SNOPT}

Case 4 repeats Case 1 but using the constrained optimization method instead of the off-design weight update method. The results for Case 4 were obtained after 44 SNOPT major iterations. The optimized solution satisfies the first-order optimality conditions for a constrained optimization problem. On-design performance has been optimized while all off-design constraints have been satisfied. As with Case 1, the performance results shown in Table 7 were obtained from regenerated meshes with the same properties as the original mesh for greater accuracy. A comparison of the performance results shown in Table 7 versus the performance results for Case 1 shown in Table 4 reveals that the on-design performance is very similar in both cases. However, there are differences in the off-design performance values. Figure 9 shows that the optimized airfoil geometries for Cases 1 and 4 are somewhat different. Further investigation is required to fully explain why two different solutions were obtained for the same optimization problem. Convergence of SNOPT is achieved with substantially less computational effort than that required using the the off-design weight update method. 


\begin{tabular}{|c|c|c|c|c|c|}
\hline Operating Point & $\alpha$ & $C_{l}$ & $C_{d}$ & $M_{\max }$ & $C_{l, \max }$ \\
\hline \hline $\mathrm{A}$ & $-1.79^{\circ}$ & 0.17 & 0.0092 & - & - \\
$\mathrm{B}$ & $-1.16^{\circ}$ & 0.28 & 0.0093 & - & - \\
$\mathrm{C}$ & $-1.21^{\circ}$ & 0.27 & 0.0102 & - & - \\
$\mathrm{D}$ & $-0.17^{\circ}$ & 0.45 & 0.0108 & - & - \\
$\mathrm{E}$ & $-1.54^{\circ}$ & 0.21 & 0.0092 & - & - \\
$\mathrm{F}$ & $-0.53^{\circ}$ & 0.36 & 0.0095 & - & - \\
$\mathrm{G}$ & $-0.65^{\circ}$ & 0.34 & 0.0104 & - & - \\
$\mathrm{H}$ & $+0.90^{\circ}$ & 0.57 & 0.0114 & - & - \\
\hline \hline $\mathrm{I}$ & $-1.19^{\circ}$ & 0.28 & 0.0108 & 1.23 & - \\
$\mathrm{J}$ & $-1.85^{\circ}$ & 0.15 & 0.0103 & 1.32 & - \\
$\mathrm{K}$ & $-0.16^{\circ}$ & 0.46 & 0.0144 & 1.31 & - \\
$\mathrm{L}$ & $-1.35^{\circ}$ & 0.25 & 0.0105 & 1.22 & - \\
$\mathrm{M}$ & $-0.21^{\circ}$ & 0.45 & 0.0149 & 1.31 & - \\
$\mathrm{N}$ & $-1.40^{\circ}$ & 0.24 & 0.0112 & 1.21 & - \\
$\mathrm{O}$ & $+1.44^{\circ}$ & 0.74 & 0.0219 & 1.35 & - \\
$\mathrm{P}$ & $-0.51^{\circ}$ & 0.40 & 0.0137 & 1.28 & - \\
\hline \hline $\mathrm{Q}$ & $+14.35^{\circ}$ & - & - & - & 1.63 \\
$\mathrm{R}$ & $+14.35^{\circ}$ & - & - & - & 1.63 \\
\hline
\end{tabular}

Table 7. Case 4 optimized airfoil performance

\section{Conclusions}

This paper presents two methods for solving practical aerodynamic design problems with competing design objectives and aerodynamic constraints using multipoint optimization. The paper addresses issues that arise in practical multipoint optimization due to the co-existence of on-design and off-design points. The application of the off-design weight update method to an eighteen-point airfoil optimization demonstrates that it is able to iteratively adjust off-design weights based on the evolution of aerodynamic performance so as to satisfy off-design aerodynamic constraints while minimizing the penalty in on-design performance. The method allows designers to identify redundant and critical operating points. Furthermore, this technique is capable of preventing the off-design constraints from being over-satisfied in order to minimize their negative influence on the on-design performance. The resulting airfoil satisfies all off-design aerodynamic constraints and has maintained overall on-design performance compared to the RAE 2822 airfoil. Moreover, virtually the same optimized airfoil can be obtained starting from the NACA 0012 airfoil. An additional optimization executed using the weight update procedure with a reduced set of off-design points confirms that the same optimized airfoil can be obtained with significantly less computational effort if redundant off-design constraints can be identified prior to performing the optimization. The second method using the constrained optimization algorithm SNOPT is capable of producing the same results as the off-design weight update method but with significantly less computational effort. A drawback to the SNOPT method is the trial and error procedure required to obtain suitable values for the parameters $\rho$ and $M_{k s}$ associated with the $K S$ function needed to achieve satisfaction of the $M_{\max }$ constraints. Generally speaking, as the number of constraints handled by the $K S$ function decreases, its conservative estimate of the maximum constraint value becomes closer to the actual maximum constraint value. This property may be exploited by investigating strategies to reduce the number of constraints, $\mathbf{g}(\mathbf{x})$, handled by the $K S$ function, thus reducing the guesswork associated with the assignment of $\rho$ and $M_{k s}$ parameters.

The concept of design under uncertainty recognizes that various uncertainties involved in the optimization procedure can lead to significant uncertainty in the optimal solution. In order to further increase the usefulness of the approach presented here, it would be beneficial to incorporate a methodology for dealing with uncertainty. 


\section{Acknowledgments}

The funding of the first and second authors by the Natural Sciences and Engineering Research Council of Canada and the third author by the Natural Sciences and Engineering Research Council of Canada and the Canada Research Chairs program is gratefully acknowledged

\section{References}

${ }^{1}$ W.K. Anderson and D.L. Bonhaus. Aerodynamic Design On Unstructured Grids for Turbulent Flows. Technical Report TM-112867, NASA, 1997.

${ }^{2}$ S. E. Cliff, J. J. Reuther, D. A. Saunders, and R. M. Hicks. Single-Point and Multipoint Aerodynamic Optimization of High Speed Civil Transport. Journal of Aircraft, 38(6), November-December 2001.

${ }^{3}$ B. Epstein, A. Jameson, S. Peigin, D. Roman, N. Harrison, and J. Vassberg. Comparative Study of 3D Wing Drag Minimization by Different Optimization Techniques. AIAA Journal, 2008.

${ }^{4}$ P. E. Gill, W. Murray, and M. A. Saunders. SNOPT: An SQP Algorithm for Large-Scale Constrained Optimization. SIAM Review, 47(1):99-131, February 2005.

${ }^{5}$ W. Li, L. Huyse, and S. Padula. Robust Airfoil Optimization to Achieve Consistent Drag Reduction Over a Mach Range. ICASE 2001-22, NASA, August 2001.

${ }^{6}$ J.R.R.A. Martins, J.J. Alonso, and J.J. Reuther. High-Fidelity Aerostructural Design Optimization of a Supersonic Business Jet. Journal of Aircraft, 41(3):523-530, 2004.

${ }^{7} \mathrm{M}$. Nemec and D. W. Zingg. Newton-Krylov algorithm for aerodynamic design using the Navier-Stokes equations. AIAA Journal, 40(6):1146-1154, June 2002.

${ }^{8}$ M. Nemec, D. W. Zingg, and T. H. Pulliam. Multipoint and multi-objective aerodynamic shape optimization. AIAA Journal, 42(6):1057-1065, June 2004.

${ }^{9}$ J. Nocedal and S. Wright. Numerical Optimization. Springer, 2nd edition, 2006.

${ }^{10}$ M.K. Poon and J.R.R.A. Martins. An Adaptive Approach to Constraint Aggregation Using Adjoint Sensitivity Analysis. Structural Multidisciplinary Optimization, 34:61-73, 2006.

${ }^{11} \mathrm{M}$ Stettner and D.P. Schrage. An Approach to Tiltrotor Wing Aeroservoelastic Optimization. AIAA/USAF/NASA/OAI, 1992. 4th Symposium on Multidisciplinary Analysis and Optimization, Cleveland, Ohio.

${ }^{12}$ G.A. Wrenn. An Indirect Method for Numerical Optimization Using the Kreisselmeier-Steinhauser Function. Technical Report CR-4220, NASA, 1989.

${ }^{13}$ D.W. Zingg and L. Billing. Toward Practical Aerodynamic Design Through Numerical Optimization. AIAA Journal, 2007.

${ }^{14}$ D.W. Zingg and S. Elias. Aerodynamic Optimization Under a Range of Operating Conditions. AIAA Journal, 44(11):2787-2792, November 2006. 


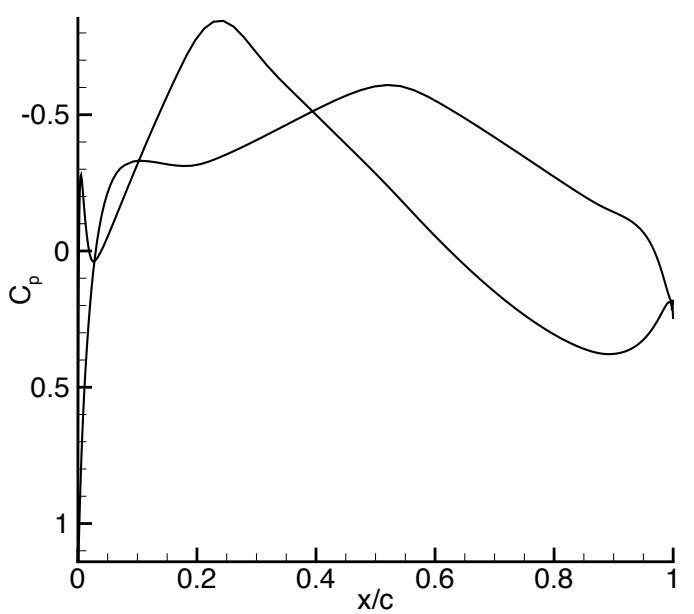

(a) Point A

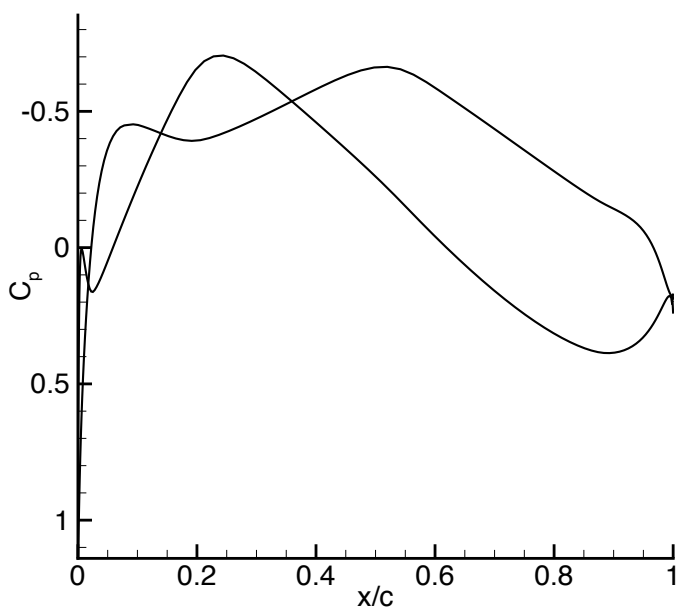

(c) Point $\mathrm{C}$

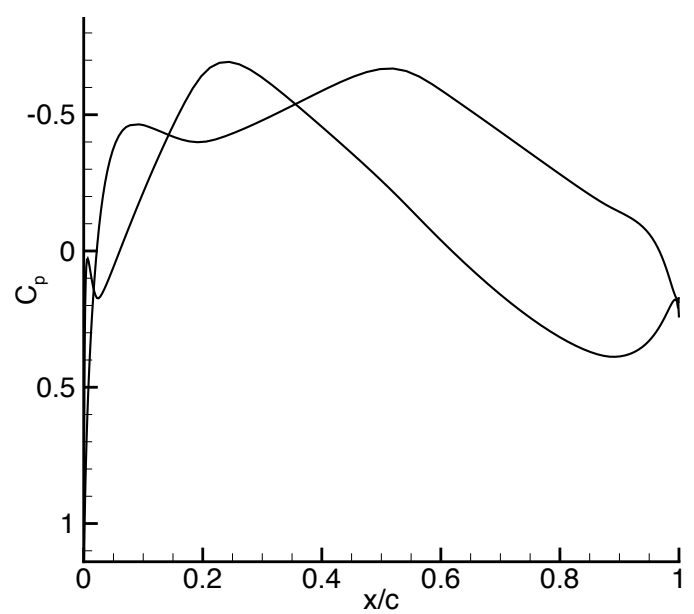

(b) Point B

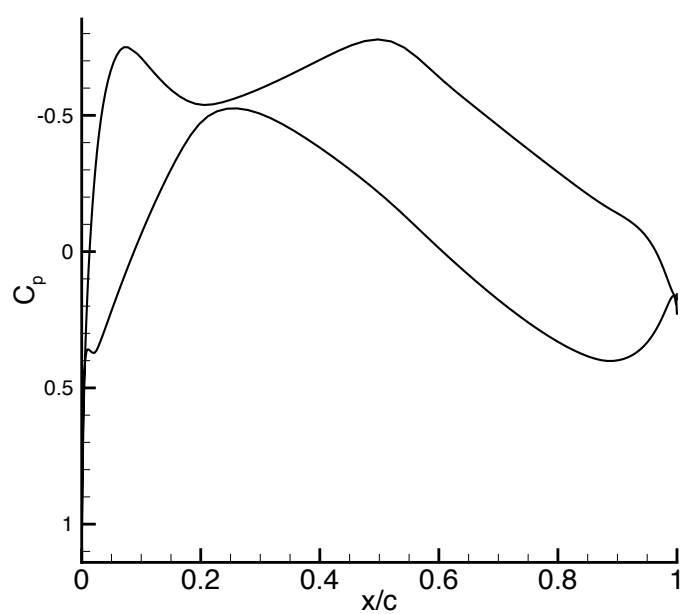

(d) Point D

Figure 10. Surface pressure coefficient distributions for operating points A-D (Case 1) 


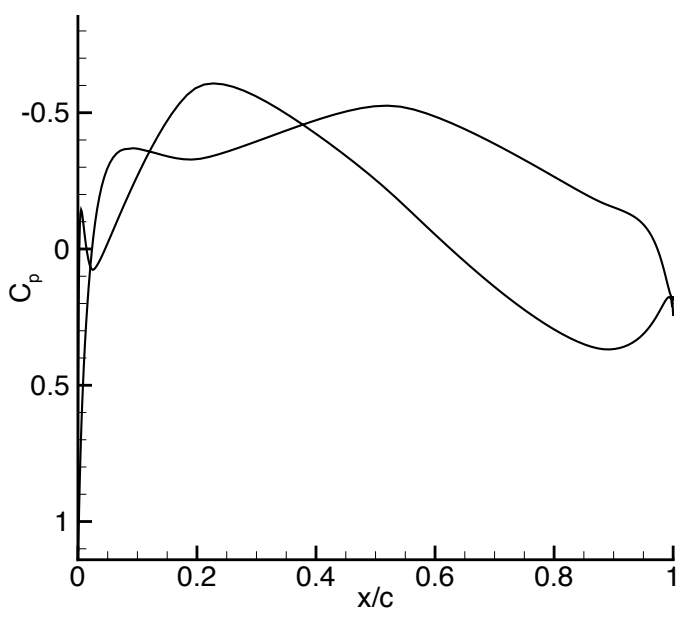

(a) Point E

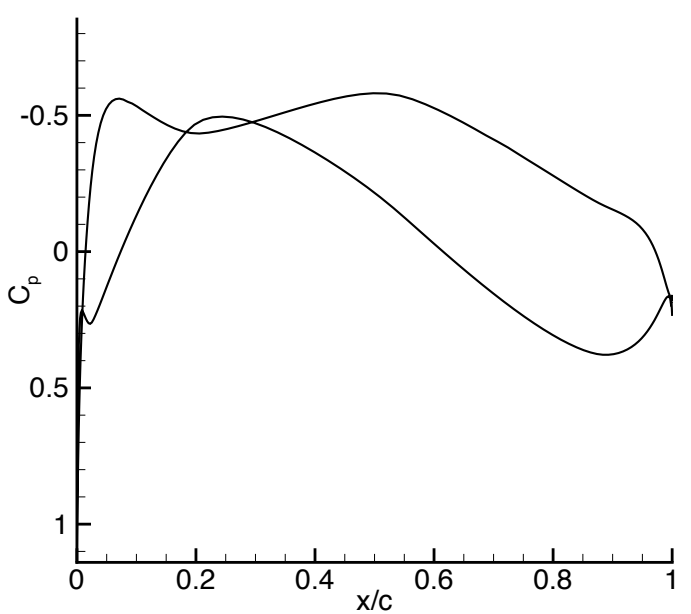

(c) Point G

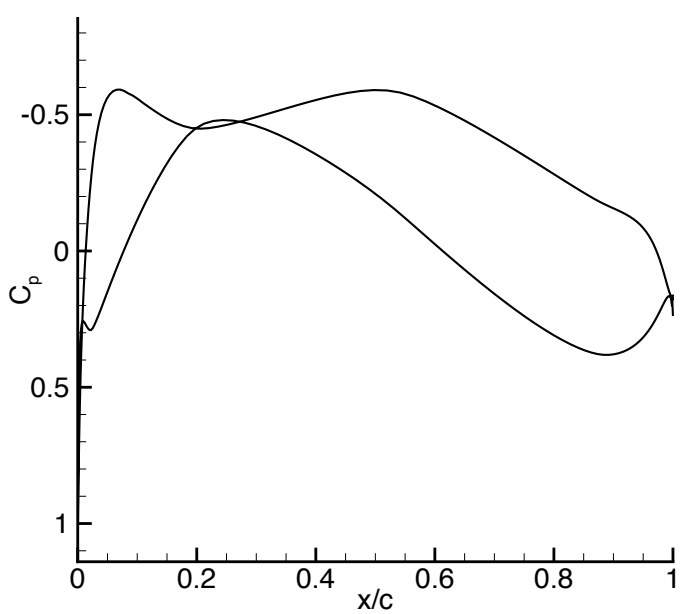

(b) Point F

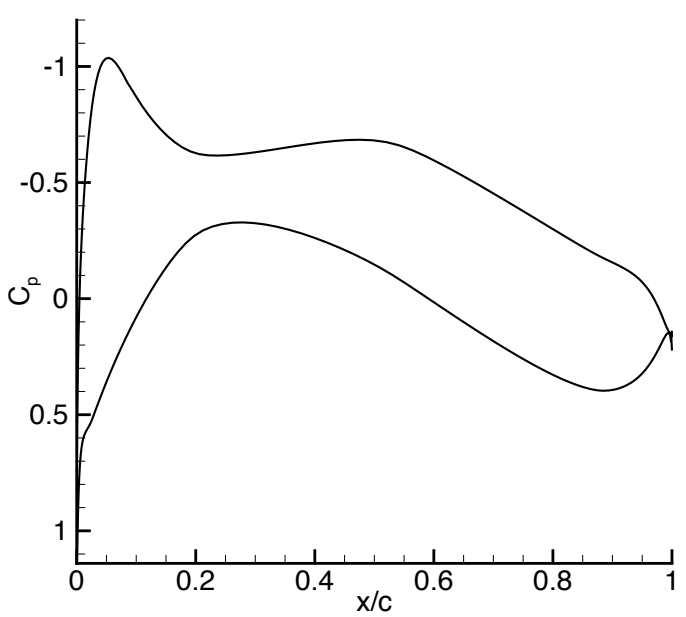

(d) Point $\mathrm{H}$

Figure 11. Surface pressure coefficient distributions for operating points E-H (Case 1) 